\section{A T2-relaxometric investigation of the brain in early psychosis: preliminary findings}

\section{S Wood', C Pantelis', P McGorry'2, D Velakoulis', G Jackson ${ }^{3}, \mathrm{G}$ Pell $^{3}$, M McConchie ${ }^{2}$, T Proffitt', G Berger ${ }^{2}$}

'Melbourne Neuropsychiatry Centre; ${ }^{2} O R Y G E N$ Research Centre; and

${ }^{3}$ Brain Research Institute, Melbourne, Australia

Despite over 15 years of research, our understanding of the brain abnormalities associated with the early phase of psychotic disorders remains rudimentary. Much effort has been put into volumetric techniques, with much variable findings. For every study that has detected abnormality in a specific brain region, there is at least one that fails to find such a difference. In part, this may be because pathology (especially in the early phase of a disorder) is not always associated with re- ductions in volume. $\mathrm{T} 2$ relaxometry, on the other hand, is an extremely sensitive (though unspecific) technique to detect brain pathology. We have studied the T2 relaxation times in the brains of 10 patients with firstepisode psychosis and seven healthy controls. We found significant reductions in T2 in several brain regions; the anterior limb of the internal capsule $[\mathrm{F}(1,15)=4.9, P=0.043]$, orbitofrontal cortex white matter $[\mathrm{F}(1,15)=4.5, P=0.051)$ and the superior temporal gyrus [gray and white matter, $\mathrm{F}(1,15)=10.9$, $P=0.005)$. Interestingly, there were no differences in the hippocampus, amygdala or dorsolateral prefrontal cortex (all $P>0.4$ ). Reductions in T2 are most likely to reflect reduced tissue water content and are strongly suggestive that edema is not a feature of the early phase of psychotic disorders. Expansion of this dataset and an examination of the relationship between $\mathrm{T} 2$ and other imaging variables are required. 